concentration of effective equivalent stratospheric chlorine (EESC) of about 30 parts per billion (p.p.b.). EESC is calculated by summing total stratospheric chlorine and bromine levels, and it quantifies their combined effect on ozone depletion in the stratosphere. The Montreal Protocol limited EESC to about 4 p.p.b., leading to a maximum total ozone loss of roughly five to six per cent.

So while the choice of a five-percent decrease in column ozone levels as the boundary for stratospheric ozone depletion appears reasonable, one could argue that a more realistic boundary is 10 or even 20 p.p.b. of EESC. Either of these boundaries would still maintain a safe distance from the 30-p.p.b. tipping point that would lead to massive ozone loss; a 10-p.p.b. EESC boundary, for example, would lead to about 15 per cent total stratospheric ozone loss.

World leaders decided to ban the industrial production of CFCs early enough that the decrease in stratospheric ozone was limited to about five per cent. Although the non-linear behaviour of lower-stratospheric ozone loss was not even a consideration in the discussions that led to the CFC ban, the decision was welljustified in light of the potential damage to human health and to ecological systems from an ozone loss greater than five per cent. It also made sense because of the CFC ban's relatively small cost to society, given that replacement compounds could be developed.

In summary, the planetary boundary concept is a very important one, and its proposal should now be followed by discussions of the connections between the various boundaries and of their association with other concepts such as the limits to growth' Importantly, this novel concept highlights the risk of reaching thresholds or tipping points for non-linear or abrupt changes in Earth-system processes. As such, it can help society to reach the agreements required for dealing effectively with existing global environmental threats, such as climate change. Stratospheric ozone depletion was properly dealt with well before crossing the boundary that would trigger an abrupt change of global proportions, but well after reaching the tipping point that caused the Antarctic ozone hole - a regional, episodic event. A five-per-cent decrease in ozone might be appropriate as a planetary boundary, but that's only true if the concept is expanded to include limits that are well within the linear regime for that Earth-system process.

Published online: 23 September 2009

doi:10.1038/climate.2009.96

Mario J. Molina is the director of the Mario Molina Center for Strategic Studies in Energy and the Environment in Mexico City and also holds a faculty position at the University of California, San Diego. e-mail:mjmolina@ucsd.edu

\title{
The devil is in the detail
}

\author{
DAVID MOLDEN
}

\section{A global limit on water consumption is necessary, but the suggested planetary boundary of 4,000 cubic kilometres per year is too generous.}

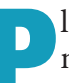
lanetary boundaries are a welcome new approach in the 'limits to growth' debate. For one thing, they shift our attention to the scale of planetary systems being altered by human activity. As a scientific organizing principle, the concept has many strengths. What scientists persistently ignore is the unpleasant fact that a good scientific concept isn't necessarily a good communications platform. In that sense, it will take much more than the presentation of a novel concept to spur action. It is imperative that we act now on several fronts to avert a calamity far greater than what we envision from climate change alone.

The key element in the planetary boundary framework is the provision of numerical target values for process variables that represent the boundaries. Rockström et al. (Nature 461, 472-475; 2009) provide first estimates for seven of nine environmental parameters by synthesizing available knowledge. It could be argued that with our limited understanding it is impossible to present reasonable numbers, or that the borders are much more malleable than the

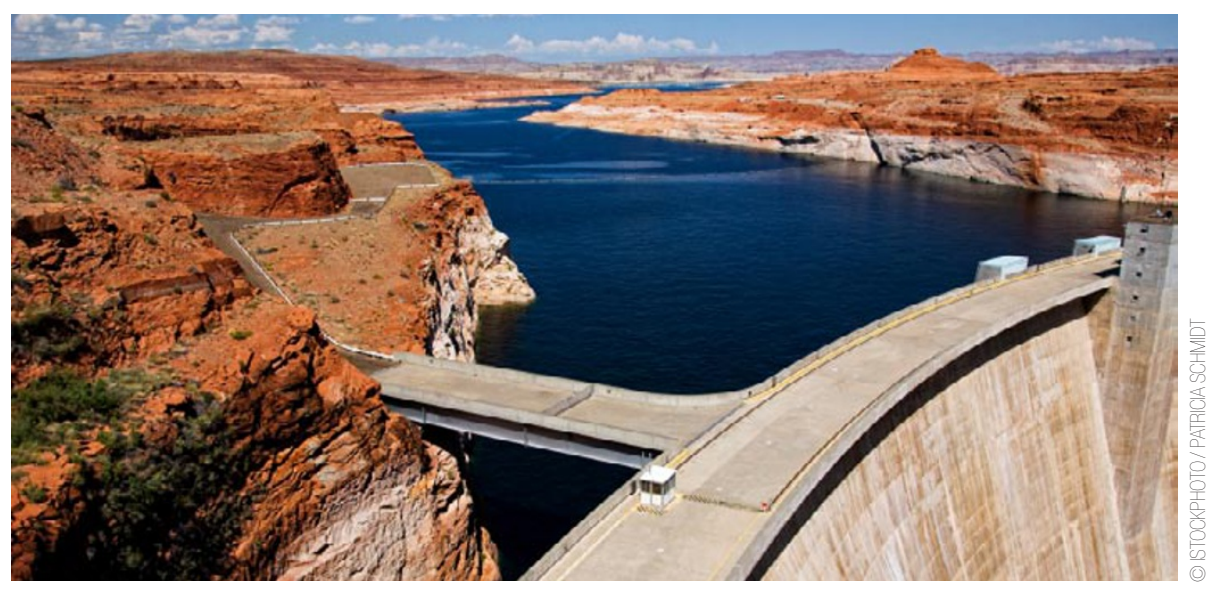

boundaries suggest, and with better or worse management, boundaries can be moved. Moreover, global values mask important issues at regional and local scales and conceal variability. On the other hand, the numbers are important because they provide targets for policymakers, giving a clear indication of the magnitude and direction of change. They also provide benchmarks and direction for science. As we improve our understanding of Earth processes and complex inter-relationships, these benchmarks can and will be updated.

So what are we to make of the water boundary suggested by the authors? Here at the International Water Management Institute, experience tells us that there are physical limits to human intervention into 
natural processes. We can also confirm that water limits have been reached or breached in many major river basins across the world, and the consequences are already manifest. For example, there is little or no additional streamflow or groundwater for further development remaining in the Murray-Darling River in Australia, the Yellow River in China, the Indus in Pakistan and India, the Amu and Syr Darya in central Asia, the Nile River, and the Colorado River in the United States and Mexico. All of these are important food-producing areas. These basins suffer from excessive pollution, river desiccation, competition for supplies and ecosystem degradation. The drying of the Aral Sea is one of the most infamous examples of ecosystem damage caused by breaching the limits of freshwater withdrawals. Freshwater biodiversity has plummeted as a result of the massive hydraulic construction era beginning in the 1960s. The main driver has been agricultural water use to meet the rising food demands of a growing population.

Johan Rockström and colleagues are suggesting that consumption of 'blue water' sources - evaporation and transpiration from rivers, lakes, groundwater reservoirs and irrigation - should not exceed 4,000 cubic kilometres per year. At present, blue water consumption is estimated at 2,600 cubic kilometres per year. The first thing to say is that the 4,000 figure is based on an analysis of a relatively small number of studies on the global supply and demand of water. When extrapolated (beyond the intentions of the original studies), they lead to a range of 4,000 to 6,000 cubic kilometres. If anything, this 4,000-cubickilometre value may well be too high.

Water for agriculture is one of the forces pushing us beyond our boundary limits. In many areas, dense concentrations of people living on arable land are using water at a rate that has exhausted supplies. In other parts of the world, there is ample water but its use is limited because the land or climate is not suitable for agriculture. In yet other places, such as sub-Saharan Africa, more water could be withdrawn, but expansion in water use is limited by financial and institutional capacity. These variations were not taken into account in the setting of the water boundary.

Another factor not taken into account is the widespread and erroneous assumption that useable water in nature can be readily accessed. In their quest for water and food security, many governments have devised grandiose plans to move massive volumes of water from water-rich to water-poor river basins. Examples include the Interlinking of Rivers Project in India and the South to North Water Diversion Project in China. The ecological consequences of these interbasin water transfers remain unclear, but they are likely to be immense.

Essentially, the concept of a global limit overlooks the importance of local conditions and the role of management in magnifying or ameliorating problems. For this reason, the water boundary suggested by Röckstrom and colleagues may be too high. That said, the planetary boundaries concept and its first estimate of numeric values give us an important warning call that must be heeded. Rather than get bogged down in detailed arguments about the weaknesses of the approach or the methods of analysis, we now have a tool we can use to help us think more deeply - and urgently - about planetary limits and the critical actions we have to take.

Published online: 23 September 2009

doi:10.1038/climate.2009.97

David Molden is the deputy director general for research at the International Water Management Institute, based near Colombo, Sri Lanka.

e-mail:d.molden@cgiar.org

\section{Consider all consequences}

\section{PETER BREWER}

\section{Ocean acidification has impacts other than simple changes in $\mathrm{pH}$, and these may need boundaries too.}

$\mathrm{n}$ their definition of planetary boundaries that humans should not transgress for fear of "deleterious or even catastrophic consequences for large parts of the world's inhabitants", Rockström et al. (Nature 461, 472-475; 2009) consider ocean acidification as an essential part of the equation. This may be true whether we consider "inhabitants" to be all life or only humans, for the ocean and its resources are deeply embedded in human culture. But the authors' suggested boundary, based on aragonite saturation - a measure of the extent to which seawater is saturated with the carbonate mineral - needs careful examination.

The term 'ocean acidification' has become the recognizable phrase to encompass the ensemble effects of elevated $\mathrm{CO}_{2}$ levels on marine life. Much as climate is understood to mean much more than temperature change, so too ocean acidification means more than simple changes in $\mathrm{pH}$. Other consequences of warming and the great $\mathrm{CO}_{2}$ invasion of the ocean also need consideration as boundaries. All aerobic life in the sea, not just calcareous animals, will be affected to some degree by the 'acidification' challenge as oxygen levels fall and carbon dioxide levels rise.

Aragonite is the most common form of calcium carbonate used by coralline animals and is the basic building block of coral reefs. Thus, it might be reasonable to expect that if we transgress the proposed boundary for ocean acidification, so that waters at increasingly shallow depths become depleted of aragonite, coral reef

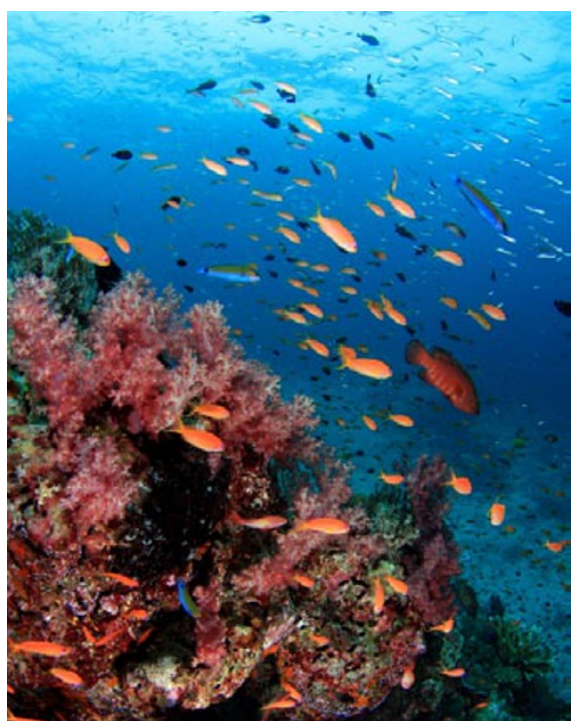

